\title{
Calculating the Number of Tunnels
}

\author{
Fajie $\mathrm{Li}^{1}$ and Reinhard Klette ${ }^{2}$ \\ ${ }^{1}$ Groningen University, The Netherlands \\ 2 The University of Auckland, New Zealand
}

\begin{abstract}
This paper considers 2-regions of grid cubes and proposes an algorithm for calculating the number of tunnels of such a region. The graph-theoretical algorithm proceeds layer by layer; a proof of its correctness is provided, and its time complexity is also given.
\end{abstract}

Keywords: Number of tunnels, digital topology, 3D grid.

\section{Introduction}

Calculations of numbers of tunnels (i.e., of the first Betti number $\beta_{1}$ ) have been a subject of interest in 3D digital topology in the context of image analysis. The Euler characteristic $\chi(K)$ of a bounded $3 \mathrm{D}$ set $K$ is given by the Poincaré formula as follows: $\chi(K)=\beta_{0}-\beta_{1}+\beta_{2}$, for Betti numbers $\beta_{0}$ (number of connected components), $\beta_{1}$ (see above), and $\beta_{2}$ (number of closed surfaces). For a review on different algorithms for calculating the Euler number, and variations in selecting the adjacency model, see [7]. See also Section 5.3.3 in [6].

Obviously, a known Euler number does not yet specify numbers $\beta_{1}$ or $\beta_{2}$, even if $\beta_{0}=1$ can be assumed. [3] proposed an incremental, theoretically efficient algorithm for calculating Betti numbers. However, now for more than 12 years, no implementation of this algorithm has been documented somewhere on the net. The authors believe that the algorithm, as proposed in this paper for calculating $\beta_{1}$, is conceptually easier to follow, and report about its implementation.

This paper presents a hierarchical method for computing the number of tunnels of a 3D binary picture (to be precise, of 2-regions of grid cubes). Images are first processed column by column (runs), then layer by layer, and finally those '2D graphs', deduced from individual layers, are merged into a single '3D graph' in order to compute the number of tunnels. The method is based on the idea of utilizing linear skeletons.

The paper starts with a theoretical part which describes how to infer a '2-D' graph from the '3-D' graphs of the layers. This is followed by a discussion of the algorithm, followed by concluding remarks.

\section{Definitions}

We apply the grid cell model [6], using 0-, 1-, 2-, or 3-cells in 3D space, which are vertices, grid edges, grid squares, or grid cubes, respectively. Two 3-cells 

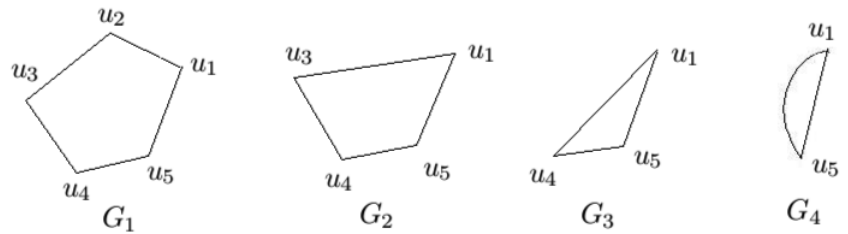

Fig. 1. $G_{1}$ is contracted into $G_{4} ; G_{4}$ can be further contracted into a loop, but not into a single node (without any edge)

(cubes, for short) $c_{1}$ and $c_{2}$ are 2-adjacent iff $c_{1} \neq c_{2}$ and the intersection $c_{1} \cap$ $c_{2}$ contains a 2-cell.

Following Section 4.2.2 of [6], the merging of two adjacent nodes $p$ and $q$ in an adjacency graph $G=[S, A]$ is defined by replacing $p$ and $q$ with a new node $r$ that is adjacent to every node in $S \backslash\{p, q\}$ to which $p$ or $q$ was originally adjacent. A finite sequence of merging operations is called a contraction; see Figure 1] If graph $G_{1}$ is contracted into $G_{2}$, then $G_{1}$ and $G_{2}$ are homeomorphic.

The linear skeleton (see Section 6.3 .3 of [6]) of a set $M \subseteq \mathbb{R}^{3}$ is defined by continuos contractions. We transform a linear skeleton into a graph by having a node at each of its singular points (see Section 7.1.3), and arcs between singular points define the edges.

Definition 1. Let $S$ be a 2-region of cubes and $G$ a graph. $G$ is a homotopic graph of $S$ iff $G$ is homeomorphic to the linear skeleton of $S$.

In Figure 2. $G_{1}$ and $G_{2}$ are both homotopic graphs of $S_{1}$, and $G_{3}$ and $G_{4}$ are both homotopic graphs of $S_{2}$.

Two relational structures $\left[S_{1}, R_{1}\right]$ and $\left[S_{2}, R_{2}\right]$ are called isomorphic iff there exists a one-to-one mapping $f$ from $S_{1}$ onto $S_{2}$ such that $p R_{1} q$ iff $f(p) R_{2} f(q)$, for all $p, q \in S_{1} . f$ is called an isomorphism.

Definition 2. Let $S$ be a 2-region and $G$ a homotopic graph of $S$. $S$ is called a minimal cubical set iff $G$ is not isomorphic to any homotopic graph of a 2-region $S^{\prime}$, where $S^{\prime}$ is obtained by removing a cube from $S$.

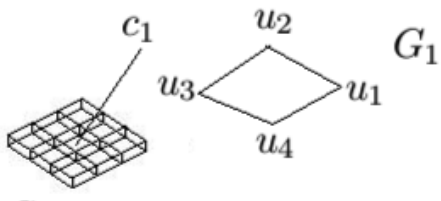

$S_{1}$

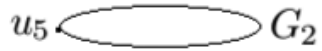

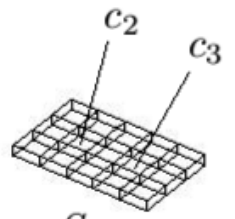

$S_{2}$
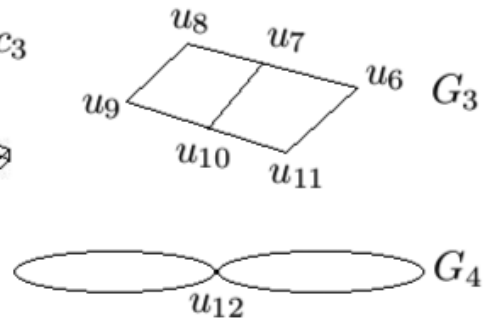

Fig. 2. Two 2-regions of cubes with homotopic graphs. $S_{1}$ does not contain cube $c_{1}$, and $S_{2}$ does not contain cubes $c_{2}$ and $c_{3}$ 
A subregion is a (non-empty) connected subset of a region. Assume a region adjacency graph and a partition of involved regions into subregions; these subregions define a subregion adjacency graph.

Definition 3. Let $L$ be a set of cubes and $H$ a homotopic graph of $L$. Let $\mathcal{R}$ be a partition of $L$ into subregions without including the infinite background component. Let $[\mathcal{R}, \mathcal{A}]$ be the subregion adjacency graph of $\mathcal{R}$. If $[\mathcal{R}, \mathcal{A}]$ is isomorphic to $H$, then $[\mathcal{R}, \mathcal{A}]$ is called a homotopic subregion adjacency graph of $L$ with respect to $H$, denoted by $\bar{H}$.

Let $f$ be the isomorphism; for the whole set $V(H)$ (The set of all vertices in $H$.), $f(V(H))$ is denoted by $\bar{V}(H)$. Figure 3 shows a layer $L$ of cubes. $H$ is a homotopic graph of $L$. Let $\bar{H}$ be a subregion adjacency graph of $L$, and $\bar{V}$ the vertices of $\bar{H}$. Each vertex $\bar{v} \in \bar{V}$ is a set of cubes.

In the rest of this paper, we denote a graph by $G=[V, E]$, where $V=V(G)$ is the set of nodes and $E$ is the set of edges. If the relation $\mathcal{A}$ (or set $E$ ) is obviously defined for a homotopic subregion adjacency graph $\bar{H}=[\mathcal{R}, \mathcal{A}]=[V, E]$ then we denote $\bar{H}$ just by $V(\bar{H})$ or $\bar{V}$.

Let $G=[V, E]$ be a graph and $V_{1} \subseteq V$. Subgraph $\left[V_{1}, E_{1}\right]$ is called the restriction of $G$ on $V_{1}$, where $E_{1}=\left\{v_{1} v_{2}: v_{1} \in V_{1} \wedge v_{2} \in V_{1}\right\}$. - The following definition is a special case of Definition 2.64 in [5].

Definition 4. Let $S$ be a family of cubical sets in $k D$ space, where $k=0,1,2,3$. Let $X$ be the set of $i$-cells contained in $S$, with $i \leq k$. Let $p, q \in X$ such that $p$ is a $j$-cell, $q$ is a $(j-1)$-cell and $p$ is the unique $j$-cell in $X$ incident with $q$, $j \leq k$. Let $X^{\prime}=X \backslash\{p, q\}$ and $S^{\prime}=\cup_{\left\{c \in X^{\prime}\right\}} c$. Then $S^{\prime}$ is called obtained from $\mathrm{S}$ via an elementary collapse of $p$ by $q$.
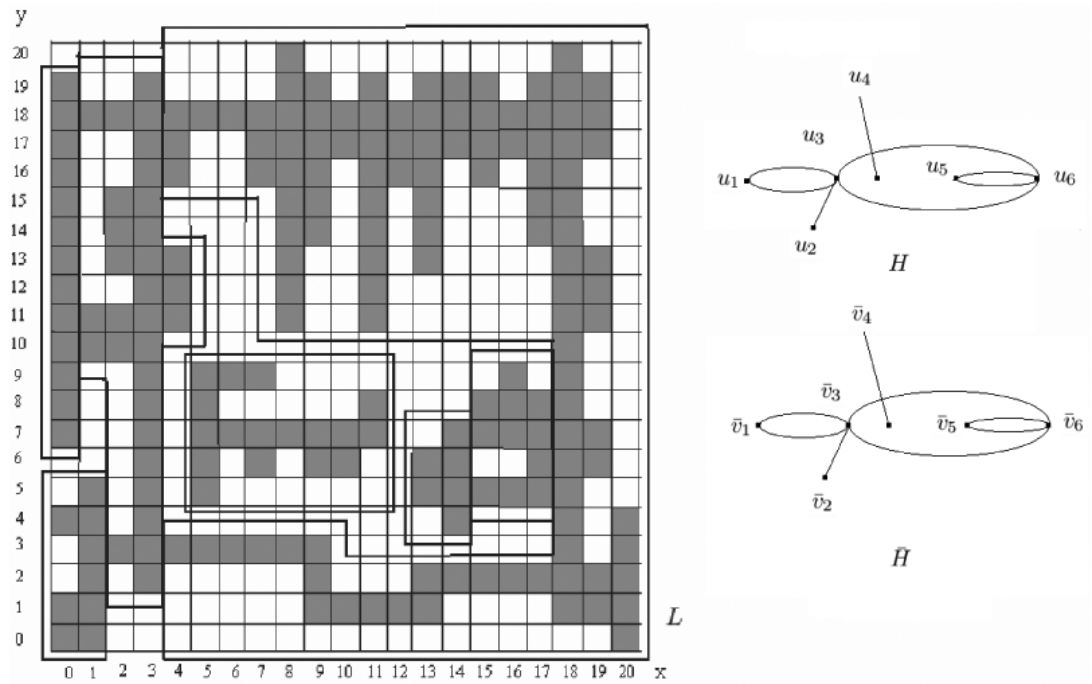

$H$

Fig. 3. A layer of cubes and a homotopic subregion adjacency graph with respect to a homotopic graph of it 

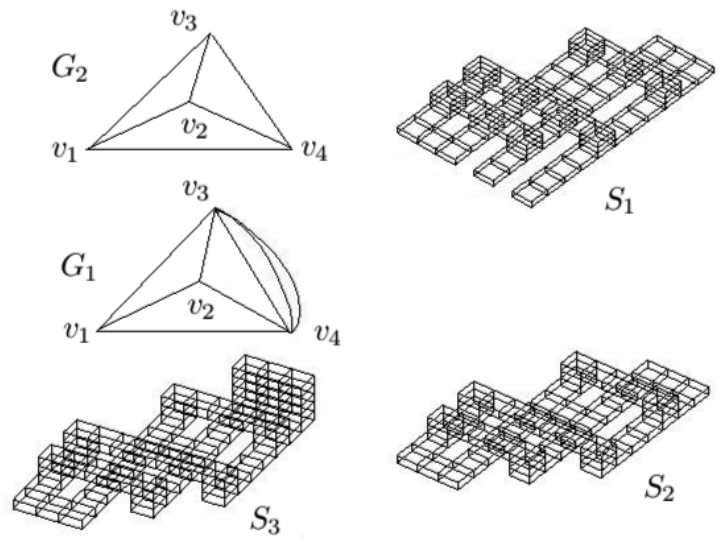

$S_{1}$

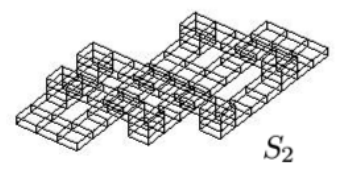

Fig. 4. A minimal cubical set and a homotopic graph

\section{Basics}

In Figure 4, $S_{2}$ is obtained from $S_{1}$ after removing 6 cubes. $S_{2}$ is a minimal cubical set and $G_{1}$ is a homotopic graph of $S_{2} . S_{3}$ is a minimal cubical set and $G_{2}$ is a homotopic graph of $S_{3}$.

Theorem 1. If a cubical 2-region $S$ is minimal such that a given graph $G$ is a homotopic graph of $S$, then we have $\beta_{1}(S)=\beta_{1}(G)$.

Corollary 1. If $S$ is a 2-connected cubical set and $G$ a homotopic graph of $S$. Then the first Betti number of $S$ is equal to the Betti number of $G$.

Let $S$ be a 2-region of cubes with nonempty intersections with grid layer $L$. Let $S_{i}$ be a component of the intersection of $S$ with layer $L, H_{i}$ a homotopic graph of $S_{i}, \bar{H}_{i}$ be a homotopic subregion adjacency graph of $S_{i}$ with respect to $H_{i}$, and let $\bar{u}_{i}, \bar{v}_{i}, \bar{w}_{i} \in \bar{H}_{i}$, for $i=1, \ldots, i_{\max }$.

Now consider a pair $\left(v_{1}, v_{2}\right)$, where $v_{i} \in V\left(H_{i}\right)$ and $\bar{v}_{i} \in V\left(\bar{H}_{i}\right)$, for $i=1,2$. Let $c$ be the number of components of $\bar{v}_{1} \cap \bar{v}_{2}$ (It is a set of 2-cells.).

Operation 1. If $c \geq 1$ then add $c$ edges between $v_{1}$ and $v_{2}$ to graph $H_{1} \cup H_{2}$. Let $E$ be the set of all edges added. Let $V_{i}$ be the set of such $v_{i} \in V\left(H_{i}\right)$. In other words, $V_{1}=\left\{v_{1}: v_{1} \in V\left(H_{1}\right) \wedge v_{1} v_{2} \in E\right\}$ and $V_{2}=\left\{v_{2}: v_{2} \in V\left(H_{2}\right) \wedge v_{1} v_{2} \in E\right\}$. Then $V_{i} \subseteq V\left(H_{i}\right)$, for $i=1,2$.

Operation 2. Let $\bar{U}_{i}$ be the restriction of $\bar{H}_{i}$ on $\bar{V}_{i}$, for $i=1$, 2. For each pair $\left(\bar{u}_{i}, \bar{w}_{i}\right)$, where $\bar{u}_{i}, \bar{w}_{i} \in \bar{U}_{i}$, let $g$ be the number of 1-paths of 2-cells $\left(p_{0}, p_{1}, \cdots, p_{n}\right)$ such that $p_{0}$ is contained in $\bar{U}_{1} \cap \bar{U}_{2}$ and $p_{n}$ is contained in $\bar{w}_{1} \cap \bar{w}_{2}$. See Figure 5 for an example.

(Note that $\bar{u}_{1} \cap \bar{u}_{2}$ and $\bar{w}_{1} \cap \bar{w}_{2}$ are also sets of 2-cells.) Delete $g$ edges between $u_{1}$ and $w_{1}$ in $H_{1}$.

Let $\left(H_{1} \cup H_{2}\right)$ be the resulting graph after applying Operations 1 and 2 on $H_{1} \cup H_{2}$. Then we have

Lemma 1. $\left(H_{1} \cup H_{2}\right)$ is a homotopic graph of the set $S_{1} \cup S_{2}$ of cubes. 

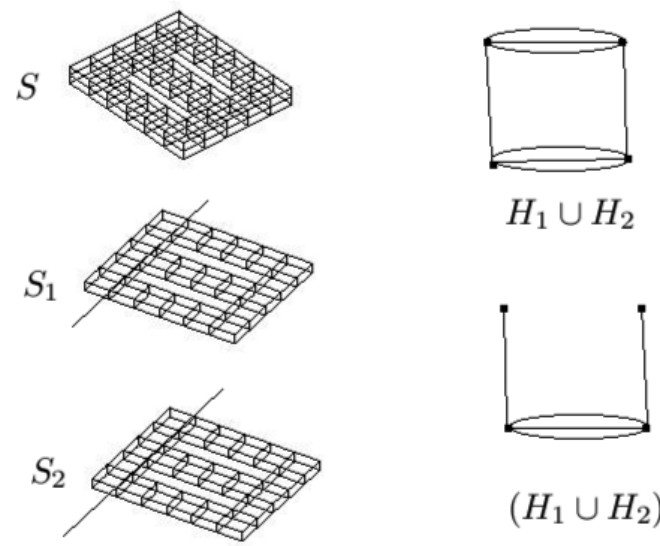

$\left(H_{1} \cup H_{2}\right)$

Fig. 5. Example with $g=3$

Let $H=\cup_{i=1}^{l} H_{i}$. Repeat Operations 1 and 2 on $H_{i} \cup H_{i+1}$ until Stop (this is guaranteed to happen, after a finite number of steps), where $i=1,2, \ldots, l-1$. Let the resulting graph be $(H)$. Then, by Lemma 1, we have the following:

Theorem 2. $(H)$ is a homotopic graph of $S$.

The following algorithm is justified by this theorem.

\section{Subprocess and Algorithm}

At first we present an algorithm for producing a homotopic subregion adjacency graph and a homotopic graph, for any given layer of cubes within the given 2-region. This is then used to describe our main algorithm.

\subsection{Preparation: Runs}

A layer of cubes can be decomposed into a number of columns (of cubes); cubes in the same column have the same $x$-coordinate. A column of cubes can then be decomposed into a number of runs (i.e., runs) of cubes. Cubes in the same run have consecutive $y$-coordinates (i.e., each run is a simple cube-arc). Each run can be represented by its two endcubes.

A run $I_{1}$ is left-(right-)adjacent to a run $I_{2}$ if the $x$-coordinate of $I_{1}$ is less (greater) than that of $I_{2}$, and there exists a cube $c_{i} \in I_{i}$ such that $c_{1} \cap c_{2}$ is a 1-cell.

Each left- or right-adjacent run of run $I$ is called a neighbor of $I$. A run-path is a sequence of runs $\left(i_{0}, i_{1}, \cdots, i_{n}\right)$ such that $i_{j+1}$ is the unique right neighbor of $i_{j}(0 \leq j \leq n-1$ and $n \geq 1) ; i_{0}$ and $i_{n}$ are called the endruns of the path. The endrun with smaller (larger) $x$-coordinate is called the first (second) endrun. A maximal run-path is a run-path such that the number of left neighbors of its 
first endrun is not one, and the number of right neighbors of its second endrun is also not one. If the number of the neighbors of the second endrun of a runpath $p_{I}$ is greater than one, then it is called the head-run of $p_{I}$, denoted by $h_{p_{I}}$. Otherwise the first endrun of $p_{I}$ is called the head-run of $p_{I}$. In both cases, we say $p_{I}$ belongs to $h_{p_{I}}$. The second endrun of $p_{I}$, different to $h_{p_{I}}$, is called the tail-run of $p_{I}$. We remove the tail-run from the run-path if the number of its neighbors is greater than one. The resulting run-path is called the reduced maximal run-path. Thus, if the number of neighbors of the tail-run is one, then the reduced maximal run-path of a run-path coincides with itself.

In Figure 3, the run in Column 18 (the cubes in this run have $x$-coordinate 18 and $y$-coordinates between 1 and 20) has 3 left neighbors and 4 right neighbors. There are two maximal run-paths from Column 3 to Column 18. The run in Column 3 is the first end- and tail-run of both, and the run in Column 18 is the second end- and the head-run of both. With the tail-run removed, they induce two reduced maximal run-paths from Column 4 to Column 18. There is also a maximal run-path from Column 5 to 11 (i.e., a disconnected "island" in the largest of the three holes). The run in column 5 is the first end- and head-run of it, and the run in Column 11 is the second end- and tail-run of it. It coincides with its reduced maximal run-path (The tail-run in Column 11 is not removed because it has only one neighbor).

\subsection{Single-Layer Subprocess}

For a single layer of cubes, do the following:

1. Get the set of all runs, denoted by $S_{I}$.

2. For each run in $S_{I}$, get and set its left and right neighbors.

3. Get the set of all maximal run-paths, denoted by $S_{P}$.

4. For each run-path in $S_{P}$, get and set its head- and tail-runs.

5. Get the set of all reduced maximal run-paths from $S_{P}$, denoted by $S_{R P}$.

6. Get the set of all runs, denoted by $S_{H}$ such that for each run $h$ in $S_{H}$, the number of neighbors of $h$ is greater than two, or the number of left neighbors of $h$ equals two and the number of right neighbors of $h$ equals zero, or the number of left neighbors of $h$ equals zero and the number of right neighbors of $h$ equals two.

7. Get the set of head-runs of all run-paths in $S_{R P}$, denoted by $S_{P H}$.

8. For each $h$ in $S_{H} \cup S_{P H}$, reset its neighbors as follows:

8.1. For each pair $h_{1}, h_{2}$ in $S_{H} \cup S_{P H}$, if they have a common neighbor $I$, remove $I$ in the set of neighbors of $h_{i}$ where the index $i$ satisfies $x_{i}<x_{i+1}(\bmod$ 2 ), where $x_{i}$ is the $x$-coordinate of $h_{i}$, for $i=1,2$.

8.2. For each $h$ in $S_{H}$, remove its neighbor if this neighbor is contained in a run-path in $S_{R P}$ (Note that 8.2 and 8.1 are independent of each other because, by definition, a run-path has at least two runs).

9. For each head-run $h$ in $S_{H} \cup S_{P H}$, let $C_{h}=C \cup C_{N} \cup C_{p}$, where $C$ is the set of all cubes contained in $h, C_{N}$ is the set of all cubes contained in neighbors of $h$, and $C_{p}$ is the set of all cubes contained in reduced maximal run-paths belonging to $h$ (i.e., $C_{P}=\left\{c: c \in p \in S_{R P} \wedge p\right.$ belongs to $\left.h\right\}$ ). 
10. Get a homotopic subregion adjacency graph $\bar{H}$ by $S_{H} \cup S_{P H}$, where each subregion is a set $C_{h}$ as obtained in Step 9;

11. Get a homotopic graph $H$ from $\bar{H}$.

We use now this single-layer subprocess for describing the main procedure, which computes the number of tunnels of a 2-region of cubes.

\subsection{Algorithm}

Assume a 2-region $S$ of cubes. We proceed as follows:

1. Decompose $S$ into layers, defined by all those $z$-coordinates with cells in $S$. (Each layer is thus the subset of $S$ defined by an identical $z$-coordinate.) Let $L_{i}$ be the $i$-th layer of $S$, where $i=1,2, \ldots, m$, and $m$ is the number of layers of $S$.

2. By the single-layer subprocess, construct a homotopic graph $H_{i}$ for each layer $L_{i}$, and a homotopic subregion adjacency graph, $\bar{H}_{i}$, with respect to $H_{i}$.

3. Without loss generality, let $L_{1}$ and $L_{2}$ be any two consecutive layers. Let $H_{1}$ or $H_{2}$ be homotopic graphs of $L_{1}$ or $L_{2}$, respectively. Let $\bar{H}_{1}$ or $\bar{H}_{2}$ be homotopic subregion adjacency graphs with respect to $H_{1}$ or $H_{2}$, respectively. For each pair $\left(v_{1}, v_{2}\right)$, where $v_{1} \in V\left(H_{1}\right), v_{2} \in V\left(H_{2}\right), \bar{v}_{1} \in V\left(\bar{H}_{1}\right)$, and $\bar{v}_{2} \in V\left(\bar{H}_{2}\right)$, do the following:

3.1. Apply the FILL algorithm (see, e.g., [6]) to find the number of components of $\bar{v}_{1} \cap \bar{v}_{2}$ (which is a set of 2-cells), denoted by $c$.

3.2. If $c \geq 1$, then add $c$ edges between $v_{1}$ and $v_{2}$ in graph $H_{1} \cup H_{2}$. Let $E$ be the set of all edges added. Let $V_{i}$ be the set of these $v_{i} \in V\left(H_{i}\right)$. In other words, $V_{1}=\left\{v_{1}: v_{1} \in V\left(H_{1}\right) \wedge v_{1} v_{2} \in E\right\}$ and $V_{2}=\left\{v_{2}: v_{2} \in V\left(H_{2}\right) \wedge v_{1} v_{2} \in E\right\}$. Then $V_{i} \subseteq V\left(H_{i}\right)$, for $i=1,2$.

3.3. Let $\bar{U}_{i}$ be the restriction of $\bar{H}_{i}$ on $\bar{V}_{i}$, where $i=1,2$. For each pair $\left(\bar{u}_{i}, \bar{w}_{i}\right)$, where $\bar{u}_{i}, \bar{w}_{i} \in \bar{U}_{i}$, apply the FILL algorithm to find the number of 1-paths of 2-cells $\left(p_{0}, p_{1}, \cdots, p_{n}\right)$ such that $p_{0}$ is contained in $\bar{u}_{1} \cap \bar{u}_{2}$, and $p_{n}$ is contained in $\bar{w}_{1} \cap \bar{w}_{2}$ (where both $\bar{u}_{1} \cap \bar{u}_{2}$ and $\bar{w}_{1} \cap \bar{w}_{2}$ are again sets of 2 -cells), denoted by $p$. Delete $p$ edges between $u_{1}$ and $w_{1}$ in $H_{1}$.

4. Apply Step 3 to every pair $L_{i}$ and $L_{i+1}$ of two consecutive layers, for $i=$ $1,2, \ldots, i_{\max }-1$. Let $G$ be the resulting graph. Then the number of tunnels of $S$ equals $E(G)-V(G)+1$. For this final step we use a basic result in combinatorial graph theory [1]: Let $G$ be a graph with $n$ nodes, $m$ edges and $k$ connected components. Then we have that $\beta_{1}(G)=m-n+k$.

\subsection{Computational Complexity}

Let $l$ be the number of cubes in the considered layer $L$, and $r$ or $c$ the number of rows or columns in $L$, respectively.

Step 1 can be computed in time $\mathcal{O}(l)$. Step 2 can be computed in $\mathcal{O}(l r)$. Step 3 can be done in $\mathcal{O}(l c)$. Analogous to Step 1, Steps 4, 5, 6 and 7 can be completed in $\mathcal{O}(l)$. Step 8.1 Step 8.2 can be finished in $\mathcal{O}\left((l r)^{2}\right)$. For Step 9, note that $\mid S_{H} \cup$ $S_{P H}|\leq| S_{I}|\leq l| C,|\leq r,| C_{N} \mid \leq r$, and $\left|C_{P}\right| \leq r c$. That means that Step 9 has 
time complexity $\mathcal{O}(\operatorname{lrc})$. Steps 10 and 11 have complexity $\mathcal{O}\left(l^{2}\right)$. In summary, a run of the single-layer subprocess has time complexity $\mathcal{O}\left((l r)^{2}\right)$.

Let $S$ be our input, a 2-region of cubes, and $i_{\max }$ the number of layers in $S$. Let $l_{i}$ be the number of cubes in the $i$ th layer of $S$, denoted by $L_{i}$. Let $r_{i}$ or $c_{i}$ be the numbers of rows or columns in layer $L_{i}$, respectively.

Step 1 can be done in $\mathcal{O}\left(i_{\max }\right)$. Step 2 was discussed above. For Step 3.1, the labeling algorithm (as, for example, in 6] ) has complexity $\mathcal{O}\left(r_{i} c_{i}\right)$. Step 3.1 has time complexity $\mathcal{O}\left(l_{i}^{2} r_{i} c_{i}\right)$. Step 3.2 can be computed in $\mathcal{O}\left(l_{i}^{2}\right)$. Step 3.3 can be completed in $\mathcal{O}\left(l_{i}^{2} r_{i}^{2} c_{i}\right)$. Step 4 calls the subprocess for all $i_{\text {max }}$ layers. Let

$$
l=\max _{1 \leq i \leq i_{\max }} l_{i}, \quad r=\max _{1 \leq i \leq i_{\max }} r_{i}, \text { and } c=\max _{1 \leq i \leq i_{\max }} c_{i}
$$

The main algorithm has time complexity $\mathcal{O}\left(i_{\max } l^{2} r^{2} c\right)$.

\section{Concluding Remarks}

[24] have developed software to compute homology. However, they are (so far) unable to inform about the computational complexity of their algorithms, and so are 58 . The latter two compute homology for any finite $k$-dimensional cubical set (no restrictions on dimensions or shapes). 2] suggests a search for improved algorithms and for new approaches to the task of homology computation while [5] leaves the problem of improving the efficiency of their algorithms to the future. [4] states that time complexity of the proposed algorithm may grow 'horrifically'.

We have presented our algorithm and analyzed its computational complexity, which is relatively low compared to other options. We also believe that the graph-theoretical nature of our algorithm is of general interest.

\section{References}

1. Bonnington, C., Little, C.: The Foundations of Topological Graph Theory. Springer, New York (1995)

2. CHomP (Atlanta) and CAPD (Kraków). Homology algorithms and software, http://www.math.gatech.edu/ chomp/homology/

3. Delfinado, C.J.A., Edelsbrunner, H.: An incremental algorithm for Betti numbers of simplicial complexes on the 3-sphere. Comput. A. Geom. Des. 12, 771-784 (1995)

4. De Silva, V.: Plex - A Matlab library for studying simplicial homology, http://math.stanford.edu/comptop/programs/plex/plexintro.pdf

5. Kaczynski, T., Mischaikow, K., Mrozek, M.: Computational Homology. Applied Mathematical Science, vol. 157. Springer, Heidelberg (2004)

6. Klette, R., Rosenfeld, A.: Digital Geometry: Geometric Methods for Digital Picture Analysis. Morgan Kaufmann, San Francisco (2004)

7. Ohser, J., Nagel, W., Schladitz, K.: The Euler number of discretized sets - on the choice of adjacency in homogeneous lattices. In: Proc. Morphology Condensed Matter. Physics Geometry Spatially Complex Systems. LNP, vol. 600, pp. 275-298 (2002)

8. Peltier, S., Alayrangues, S., Fuchs, L., Lachaud, J.: Computation of homology groups and generators. In: Andrès, É., Damiand, G., Lienhardt, P. (eds.) DGCI 2005. LNCS, vol. 3429, pp. 195-205. Springer, Heidelberg (2005) 